\title{
Analytical integrations of the linearly interpolated triangular IBEM element with the aid of the Matlab Symbolic Math Toolbox
}

\author{
Gong Andong \\ School of Geosciences and Info-physics, \\ Central South University, P. R. China
}

\begin{abstract}
This paper deals with the analytical integration over the linearly interpolated triangular boundary element involved in an indirect boundary element method (IBEM) for solving three dimensional potential problems. The analytical solutions of all the IBEM integrals are derived using the Matlab Symbolic Math Toolbox, with most of the deriving works carried out by computer, and accompanied by a few crucial manual rectifications and integrating techniques. Compared with the presently published kindred analytical solutions, the computer-produced and human-rectified ones take much less tedious manual work and have their own advantages in implementation, such as convenience for programming, and no need to trace the relevant literature for detailed expressions, etc. A calculation test is also carried out to justify the analytical solutions.

Keywords: indirect boundary element method, triangular element, analytical integration, Matlab Symbolic Math Toolbox.
\end{abstract}

\section{Introduction}

The boundary element method (BEM) plays an important role in the solution of the potential problems which are governed by the Laplace equation or Poisson equation owing to its much lower requirement for computer memory and obvious superiority in solving infinite-boundary problems. Depending on the boundary integral equations (BIE) applied, BEM can be divided into direct boundary element method (DBEM) and indirect boundary element method (IBEM). The former uses the second Green formula as BIE, while the latter uses 
the convolution of a density function and the Laplace equation's fundamental solution. A key step of BEM is to evaluate the piecewise boundary element integrals appearing in the integral equations (refer to [1]). When using linearly interpolated triangular elements, the integrals appearing in the DBEM make up the subset of those appearing in the IBEM, so in this paper, dealing with the IBEM integrals is enough to cover all the relevant BEM integrals.

In two-dimensional problems, exactly evaluating the boundary element integrals is not a hard task, since the closed-form analytical solutions of the integrals on a linear boundary element can be easily derived [2-5]. In threedimensional problems, however, analytical solutions of the integrals cannot be easily obtained and is usually replaced by numerical integration, such as Gaussian quadrature [6-8]. But unlike the analytical solutions mentioned above, the numerical methods usually have a trade-off between the accuracy and the efficiency of the calculation, i.e., to get the higher accuracy, the longer computational time has to be consumed, especially when the singular point is on the boundary or near to the boundary. To obtain the closed-form analytical integrations over the triangular elements for three-dimensional potential problems, a lot of research has been carried out, and some important progresses have been made. For example [6, 9, 10] provide several sets of analytical solutions of the integrals for three-dimensional linearly interpolated triangular element, as well as instructive methods for deducing the analytical solutions. Nevertheless, their solutions are intended for DBEM, thus having no analytical solutions for the integrals uniquely appearing in IDBEM, and they can only be applied to triangular elements with linearly interpolated potential and constant flux, other than linearly interpolated potential and flux. Furthermore, the solutions involve lots of spatial geometric factors of both the element itself and its position relative to the fixed point, making computer programming more difficult and less robust. Salvadori and Temponi [11] and Salvadori [12] provide a set of analytical solutions for both CPV integrals and HFP integrals; however, the solutions appear to be very complex and are distributed in several different articles, which makes the practical application difficult.

In this paper, a completely new set of analytical solutions of the integrals on linearly interpolated triangular element is derived for three-dimensional IBEM. Compared with those in [6, 9-12] the solutions are in closed form alike, but have the following characteristics: First, being derived with the software Matlab Symbolic Math Toolbox, most of the analytical expressions are automatically produced and processed with a computer. Second, as the toolbox introduces the square root of -1 when deriving the analytical solutions for some of the integrals, the evaluation of their analytical expressions involves complex calculation, although the final result of the evaluation is a real number, and the last, a few manual rectifications and integration techniques are necessary.

The paper is organized as follows: general formulae are presented in Section 2; analytical integrations for electric displacement integrals are derived in Section 3; analytical integrations for potential integrals are deduced in Section 4; and Section 5 presents a calculation example to justify the proposed analytical solutions; the conclusion is presented in Section 6 . 


\section{General formulae}

Potential problems include electrostatic, gravitational, and steady-state heat conducting problems, etc. Without loss of generality, we take the electrostatic problem as example in this paper. In a typical three-dimensional electrostatic problem, the Laplace equation can be written as

$$
\nabla^{2} u(x, y, z)=0,
$$

which governs the potential $u$ in a three-dimensional domain $\Omega$ enclosed by a boundary surface $\Gamma^{\prime}$. Suppose that $\Gamma^{\prime}$ is enclosed by a virtual boundary surface $\Gamma$, and there is an interval between the two surfaces. $\Gamma$ is divided into a certain number of triangular elements, with neighboring elements having two common vertexes, the standard IBEM procedure leads to the following equation:

$$
\left\{\begin{array}{l}
u_{j}=\frac{1}{4 \pi} \sum_{n=1}^{N_{e}} \int_{\Delta_{n}} \bar{q} \frac{1}{r} d \Gamma \\
\vec{D}_{j}=\frac{1}{4 \pi} \sum_{n=1}^{N_{e}} \int_{\Delta_{n}} \bar{q} \frac{\vec{r}}{r^{3}} d \Gamma
\end{array}\right.
$$

where $N_{e}$ is the total number of the elements on $\Gamma, \Delta_{\mathrm{n}}$ denotes the integration area of the nth element, $u_{j}$ denotes the potential value at collocation point $P_{j}, \vec{D}_{j}$ denotes the electric displacement vector at $P_{j}, \bar{q}$ denotes the linearly interpolated density of the source charge on the element, and $\vec{r}$ is the spatial vector from the source point on the element to the collocation point $P_{j}$.

For a single triangular element, the three-dimensional Cartesian element coordinate system is established as shown in Figure 1. The triangular element ${ }_{\triangle} P_{1} P_{2} P_{3}$ is in the $\mathrm{xOy}$ plane, with $P_{1}$ coinciding with the origin $O$. The coordinate system is established so that the three components $x_{1}, y_{1}$ and $y_{3}$ are 0 's, thereby decreases the complexity of the final analytical solutions. Note the conditions $y_{2}>0, x_{2}>0, x_{3}>0$ and $x_{2} \neq x_{3}$ must be satisfied in the coordinate system.

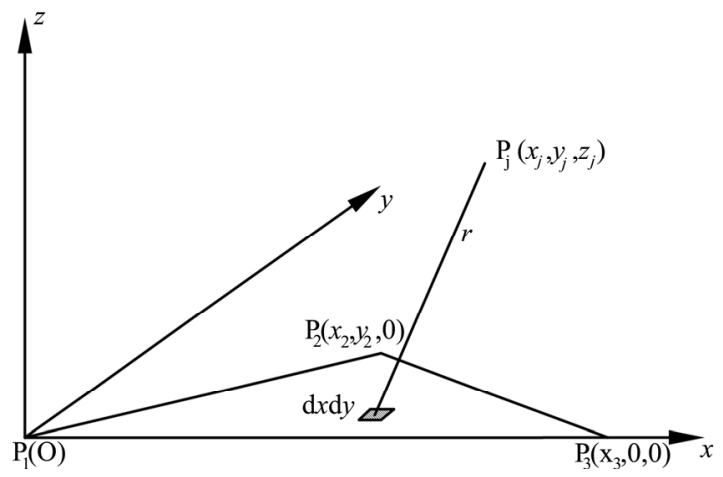

Figure 1: Element coordinate system. 
In the triangular element shown in Figure 1, the source charge density $\bar{q}$ are linearly interpolated as below:

where

$$
\bar{q}(x, y)=N_{1} q_{1}+N_{2} q_{2}+N_{3} q_{3},
$$

$$
N_{1}=1-\frac{x}{x_{3}}+\frac{\left(x_{2}-x_{3}\right) y}{y_{2} x_{3}}, N_{2}=\frac{y}{y_{2}}, N_{3}=\frac{x}{x_{3}}-\frac{x_{2} y}{y_{2} x_{3}},
$$

are the linear shape functions. Substitute (3) into (2), and after algebraic manipulations, we have

$$
\left\{\begin{array}{c}
u_{j}=\frac{1}{4 \pi} \sum_{n=1}^{N_{e}} \int_{\Delta_{n}}\left(a_{n}^{q}+b_{n}^{q} x+c_{n}^{q} y\right) \frac{1}{r} d x d y \\
D_{j x}=\frac{1}{4 \pi} \sum_{n=1}^{N_{e}} \int_{\Delta_{n}}\left(a_{n}^{q}+b_{n}^{q} x+c_{n}^{q} y\right) \frac{x_{j}-x}{r^{3}} d x d y \\
D_{j y}=\frac{1}{4 \pi} \sum_{n=1}^{N_{e}} \int_{\Delta_{n}}\left(a_{n}^{q}+b_{n}^{q} x+c_{n}^{q} y\right) \frac{y_{j}-y}{r^{3}} d x d y \\
D_{j z}=\frac{1}{4 \pi} \sum_{n=1}^{N_{e}} \int_{\Delta_{n}}\left(a_{n}^{q}+b_{n}^{q} x+c_{n}^{q} y\right) \frac{z_{j}}{r^{3}} d x d y
\end{array}\right.
$$

where $r=\sqrt{\left(x_{j}-x\right)^{2}+\left(y_{j}-y\right)^{2}+z_{j}^{2}}$, denoting the distance between $(x, y)$ on the element and $P_{j}\left(x_{j}, y_{j}, z_{j}\right)$ (see Figure 1$), \mathrm{D}_{\mathrm{j} x}, \mathrm{D}_{\mathrm{jy}}$, and $\mathrm{D}_{\mathrm{jz}}$ are the three components of the electric displacement movement vector. For an element, the expressions of the coefficients $a^{q}, b^{q}$, and $c^{q}$ are as follows:

$$
a^{q}=q_{1} ; b^{q}=\frac{q_{3}-q_{1}}{x_{3}} ; c^{q}=\frac{\left(x_{2}-x_{3}\right) q_{1}+x_{3} q_{2}-x_{2} q_{3}}{x_{3} y_{2}} .
$$

To proceed with the IBEM, the next step is to calculate the following integrals which are extracted from (4):

$$
\begin{gathered}
G_{k}=\int_{0}^{y_{2}} \int_{\frac{x_{2} y}{y_{2}}}^{x_{3}+\frac{\left(x_{2}-x_{3}\right) y}{y_{2}}} \frac{A}{\sqrt{\left(x_{j}-x\right)^{2}+\left(y_{j}-y\right)^{2}+z_{j}^{2}}} d x d y \\
H_{l}=\int_{0}^{y_{2}} \int_{\frac{x_{2} y}{y_{2}}}^{x_{3}+\frac{\left(x_{2}-x_{3}\right) y}{y_{2}}} \frac{A B}{\left[\left(x_{j}-x\right)^{2}+\left(y_{j}-y\right)^{2}+z_{j}^{2}\right]^{1.5}} d x d y
\end{gathered}
$$

where $A \in\{1, \mathrm{x}, \mathrm{y}\}, \mathrm{B} \in\left\{\mathrm{x}_{\mathrm{j}}-\mathrm{x}, \mathrm{y}_{\mathrm{j}}-\mathrm{y}, \mathrm{z}_{\mathrm{j}}\right\}, \mathrm{k}=1$ to 3 , and $\mathrm{l}=1$ to 9 . So (5) and (6) represent twelve integrals altogether. The three integrals represented by (5) are named potential integrals and the three integrals represented by (6) are named electric displacement integrals in this paper, since they are used to calculate the potential and the electric displacement at $P_{j}$ respectively.

\section{Analytical integrations for the electric displacement integrals}

Using the Symbolic Math Toolbox of Matlab, the analytical solutions for the integrals in (6) can be obtained in the form of symbolic strings. Here we take the integral 


$$
H=\int_{0}^{y_{2}} \int_{\frac{x_{2} y}{y_{2}}}^{x_{3}+\frac{\left(x_{2}-x_{3}\right) y}{y_{2}}} \frac{y}{\left[\left(x_{j}-x\right)^{2}+\left(y_{j}-y\right)^{2}+z_{j}^{2}\right]^{1.5}} d x d y
$$

as a representative example to demonstrate the detailed integration method.

First, run the following Matlab codes in the Matlab command window, where the italic text following the symbol ' $\%$ ' is the comment for the code in the next line.

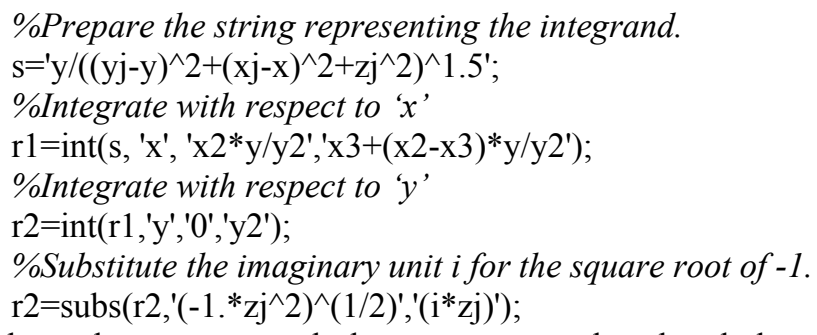

after the codes are executed, the computer-produced and closed-form analytical solution can be found in the string-type variable $r 2$. Using a few string processing functions in the Matlab symbolic toolbox, it can be simplified to a much shorter string. However, if we evaluate the solution now, we will find that it may produce distorted result. Figure 2 is the continuously evaluated result of $\mathrm{r} 2$ illustrated using the Matlab plot function, with $\mathrm{x}_{\mathrm{j}}=0$ to $3, \mathrm{y}_{\mathrm{j}}=0.4, \mathrm{z}_{\mathrm{j}}=0.21$, $\mathrm{x}_{2}=2.5, \mathrm{y}_{2}=0.866, \mathrm{x}_{3}=4$. From Figure 2(a) which illustrates the directly evaluated $\mathrm{r} 2$, it can be seen that the plotted curve is not continuous, and there are two step-changes near the points $\mathrm{x}_{\mathrm{j}}=0.75$ and $\mathrm{x}_{\mathrm{j}}=1.5$ respectively, which distort the evaluation.

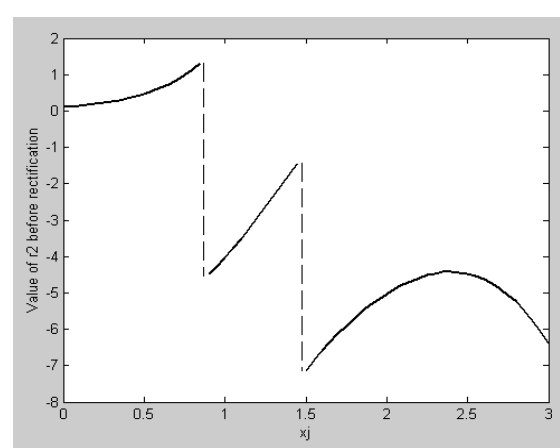

(a)

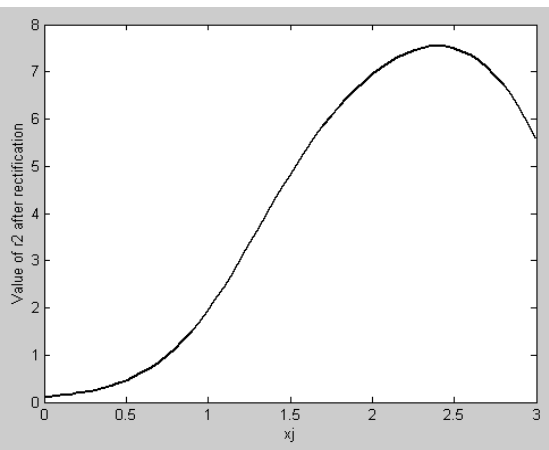

(b)

Figure 2: The evaluated result of the analytical solution before and after rectification.

After a lot of calculation trials, we find that the distortion is caused by the $\operatorname{atanh}()$ function in the $\mathrm{r} 2$, for the reason that every time a step-change happens during the process of the continuous evaluation, a sign shift between '+' and '-' occurs on the imaginary part of the $\operatorname{atanh}()$ function's output value. Therefore, 
one of the approach to avoid the distortion is to keep the imaginary part positive using the following procedure:

$$
\operatorname{atanh}(b) \rightarrow \text { a; if } \operatorname{imag}(a)<0 \text { then }(a+\pi \text { i) } \rightarrow \text { a; }
$$

where the variables $a$ and $b$ are of complex type, representing the output and input values of the atanh() function, and the function imag() takes the imaginary part of a. After the rectification, the final expression of the analytical solution is given below:

$$
H=\operatorname{real}\left[\left(y_{j}+z_{j} \mathrm{i}\right)\left(p_{23}+p_{24}\right)\right]+\left[\frac{x_{3}-x_{2}}{\sqrt{p_{8}}}\left(p_{22}-p_{20}\right)+\frac{x_{2}}{\sqrt{p_{7}}}\left(p_{21}-p_{19}\right)\right],
$$

where the function real() takes the real part of the input complex value, and the analytical expressions of $p_{n}$ in (7) and other parts of the paper are listed in Appendix A.

The analytical solutions for the other 8 electric displacement integrals have also been derived using the Matlab Symbolic Math Toolbox, and their deriving method and rectification method are generally the same as the above, for the sake of concision, they are not listed in this paper.

\section{Analytical integrations for the potential integrals}

Unlike the integrals in (6), the analytical solutions of those in (5) cannot be obtained by calling the int() function twice. To get their analytical solution using Matlab, some integration techniques must be used manually.

\subsection{Inner integrations for $G_{1}, G_{2}$ and $G_{3}$}

For the three integrals in (5), first perform the inner integrations with respect to $x$ using the Matlab's int() function, and we have:

$$
\begin{gathered}
G_{1}=\int_{0}^{y_{2}} \log \left[\frac{x_{2}-x_{3}}{y_{2}} y+\left(x_{3}-x_{j}\right)+\sqrt{\frac{p_{8}}{y_{2}^{2}} y^{2}+\frac{2 p_{10}}{y_{2}} y+p_{13}}\right] d y, \\
-\int_{0}^{y_{2}} \log \left(\frac{x_{2}}{y_{2}} y-x_{j}+\sqrt{\frac{p_{7}}{y_{2}^{2}} y^{2}+\frac{2 p_{9}}{y_{2}} y+p_{14}}\right) d y \\
G_{2}=x_{j} G_{1}+\int_{0}^{y_{2}}\left(\sqrt{\frac{p_{8}}{y_{2}^{2}} y^{2}+\frac{2 p_{10}}{y_{2}} y+p_{13}}-\sqrt{\frac{p_{7}}{y_{2}^{2}} y^{2}+\frac{2 p_{9}}{y_{2}} y+p_{14}}\right) d y, \\
G_{3}=\int_{0}^{y_{2}} \log \left[\frac{x_{2}-x_{3}}{y_{2}} y+\left(x_{3}-x_{j}\right)+\sqrt{\frac{p_{8}}{y_{2}^{2}} y^{2}+\frac{2 p_{10}}{y_{2}} y+p_{13}}\right] y d y \\
-\int_{0}^{y_{2}} \log \left(\frac{x_{2}}{y_{2}} y-x_{j}+\sqrt{\frac{p_{7}}{y_{2}^{2}} y^{2}+\frac{2 p_{9}}{y_{2}} y+p_{14}}\right) y d y
\end{gathered}
$$

From (8) to (10), it can be seen that $G_{1}, G_{2}$ and $G_{3}$ are linear combinations of the following three types of integrals:

$$
\int_{0}^{y_{2}} \log \left(a y+b+\sqrt{c y^{2}+d y+e}\right) d y,
$$




$$
\begin{gathered}
\int_{0}^{y_{2}} \log \left(a y+b+\sqrt{c y^{2}+d y+e}\right) y d y, \\
\int_{0}^{y_{2}}\left(\sqrt{c y^{2}+d y+e}-\sqrt{\dot{c} y^{2}+d^{\prime} y+\dot{e}}\right) d y,
\end{gathered}
$$

where the $a, b, c, d, e$ and $c^{\prime}, d^{\prime}, e^{\prime}$ represent the constant real coefficients shown in (8) and (9), and these coefficients depend on the coordinates of the element vertexes and the point $P_{j}$ (see Figure 1). So, it is essential to find the analytical solutions of the three types of integrals.

\subsection{Analytical solutions of the integrals represented by (11)}

One of the integrals represented by (11) is

$$
\int_{0}^{y_{2}} \log \left[\frac{x_{2}-x_{3}}{y_{2}} y+\left(x_{3}-x_{j}\right)+\sqrt{\frac{p_{8}}{y_{2}^{2}} y^{2}+\frac{2 p_{10}}{y_{2}} y+p_{13}}\right] d y .
$$

Let

$$
t(y)=\frac{x_{2}-x_{3}}{y_{2}} y+\left(x_{3}-x_{j}\right)+\sqrt{\frac{p_{8}}{y_{2}^{2}} y^{2}+\frac{2 p_{10}}{y_{2}} y+p_{13}},
$$

substitute it into (14), and perform integration by part, then we have:

$$
\int_{0}^{y_{2}} \log (t) d y=\left[y(t) \log (t)-\int \frac{y(t)}{t} d t\right]_{t(0)}^{t\left(y_{2}\right)} .
$$

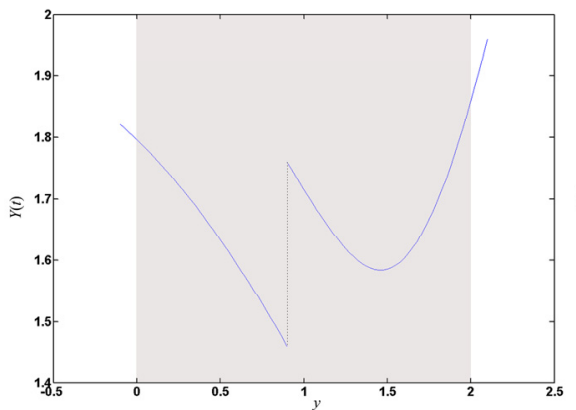

(a)

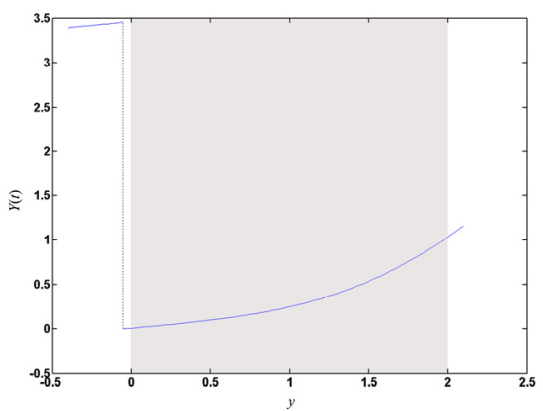

(b)

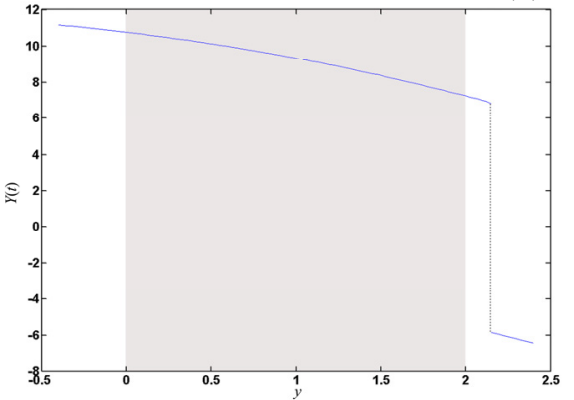

(c)

Figure 3: Three possible positions of $\left[0, y_{2}\right]$ relative to the joint of $Y(t)$. (The grayed region illustrates the interval $\left[0, y_{2}\right]$.) 
where the part in the square bracket is denoted with $Y(t)$, i.e.,

$$
Y(t)=y(t) \log (t)-\int \frac{y(t)}{t} d t .
$$

Solve the equation of (15) for $y$, and the following two expressions are obtained:

$$
\left\{\begin{array}{l}
y_{I}(t)=y_{j}+\frac{x_{3}-x_{2}}{y_{2}} t+\frac{\sqrt{p_{8} t^{2}+2 y_{2} p_{11} t-z_{j}^{2} y_{2}^{2}}}{y_{2}} \\
y_{I I}(t)=y_{j}+\frac{x_{3}-x_{2}}{y_{2}} t-\frac{\sqrt{p_{8} t^{2}+2 y_{2} p_{11} t-z_{j}^{2} y_{2}^{2}}}{y_{2}}
\end{array}\right.
$$

Substitute them into (17) separately and solve the indefinite integrals, then we have two analytical expressions for $Y(t)$, which are shown below:

$$
\begin{aligned}
& Y_{I}(t)=\left[y_{I}(t)-y_{j}\right][\log (t)-1]-\frac{p_{26} p_{11}}{\sqrt{p_{8}}}+\left|z_{j}\right| \operatorname{atan}\left(\frac{p_{11} t-z_{j}^{2} y_{2}}{\left|z_{j}\right| \sqrt{p_{25}}}\right), \\
& Y_{I I}(t)=\left[y_{I I}(t)-y_{j}\right][\log (t)-1]+\frac{p_{26} p_{11}}{\sqrt{p_{8}}}-\left|z_{j}\right| \operatorname{atan}\left(\frac{p_{11} t-z_{j}^{2} y_{2}}{\left|z_{j}\right| \sqrt{p_{25}}}\right) .
\end{aligned}
$$

Since $t$ is a function of $y, Y(t)$ is also a function of $y$, and it is expressed with $Y_{I}(t)$ and $Y_{I I}(t)$ conditionally and sectionally, i.e., for an arbitrary $y$,

$$
Y(t)=\left\{\begin{array}{ll}
Y_{I}(t) & \text { if } y=y_{I}[t(y)] \\
Y_{I I}(t) & \text { if } y=y_{I I}[t(y)]
\end{array} .\right.
$$

The graph of $Y(t)$ vs. $y$ can be plotted in accordance with (21), and the plotted curve is generally continuous and smooth, except for a step change at the joint of $Y_{I}(t)$ and $Y_{I I}(t)$.Thus, to evaluate the $[Y(t)]_{t(0)}^{\left.t y_{2}\right)}$ in (16), three possible positions of the interval $\left[0, y_{2}\right]$ relative to the joint point $y_{s}$ have to be considered (see Figure 3 where all curves are plotted with $y_{2}=2$ ):

If the interval $\left[0, y_{2}\right]$ is covered by $Y_{I}(t)$ as shown in Figure 3(a),

$$
\int_{0}^{y_{2}} \log (t) d y=[Y(t)]_{t(0)}^{t\left(y_{2}\right)}=Y_{I}\left[t\left(y_{2}\right)\right]-Y_{I}[t(0)]
$$

if $\left[0, y_{2}\right]$ is covered by $Y_{I I}(t)$ as shown in Figure $3(\mathrm{~b})$,

$$
\int_{0}^{y_{2}} \log (t) d y=[Y(t)]_{t(0)}^{t\left(y_{2}\right)}=Y_{I I}\left[t\left(y_{2}\right)\right]-Y_{I I}[t(0)] ;
$$

in the case that the joint point $y_{s}$ is within $\left[0, y_{2}\right]$, as shown in Figure 3(c), the step change has to be eliminated, and we have

$$
\int_{0}^{y_{2}} \log (t) d y=[Y(t)]_{t(0)}^{t\left(y_{2}\right)}=Y_{\mathrm{I}}\left[t\left(y_{2}\right)\right]-Y_{\mathrm{II}}[t(0)]-S,
$$

where $S=Y_{I}\left[t\left(y_{s}\right)\right]-Y_{I I}\left[t\left(y_{s}\right)\right]$, denoting the step change at $y_{s}$. Now the problem is how to evaluate the $y_{s}$ which denotes the position of the joint point. It can be proved that the $y_{s}$ has the following three properties:

$$
\left.\cdot \frac{d t(y)}{d y}\right|_{y=y_{s}}=0 \text {; II . } y_{I}\left[t\left(y_{s}\right)\right]=y_{I I}\left[t\left(y_{s}\right)\right] \text {; III. } \sqrt{p_{8} t\left(y_{s}\right)^{2}+2 y_{2} p_{11} t\left(y_{s}\right)-z_{j}^{2} y_{2}^{2}}=0 \text {. }
$$

From the property I, we know the $y_{s}$ must satisfy the following equality:

$$
\sqrt{p_{8} y_{s}^{2} / y_{2}^{2}+2 p_{10} y_{s} / y_{2}+p_{13}}=\frac{p_{8} y_{s} / y_{2}+p_{10}}{x_{3}-x_{2}},
$$


solve (25) for $y_{s}$, and we have the following two expressions:

$$
\left\{\begin{array}{l}
y_{s 1}=\frac{-p_{10} y_{2}+\left|x_{3}-x_{2}\right| \sqrt{p_{8} z_{j}^{2}+p_{11}^{2}}}{p_{8}} . \\
y_{s 2}=\frac{-p_{10} y_{2}-\left|x_{3}-x_{2}\right| \sqrt{p_{8} z_{j}^{2}+p_{11}^{2}}}{p_{8}} .
\end{array}\right.
$$

Since there is only one joint point, it is necessary to distinguish the appropriate expression from the other. By observing (25), we find the appropriate $y_{s}$ must satisfy the inequality below, while the other must not,

$$
\frac{p_{8} y_{s} / y_{2}+p_{10}}{x_{3}-x_{2}}>0 \text {. }
$$

and (27) is used as the criterion for selecting the appropriate expression for $y_{s}$.

Besides, according to the properties II and III, the evaluation of the step change $S$ in (24) can be simplified as below:

$$
S=Y_{I}\left[t\left(y_{s}\right)\right]-Y_{I I}\left[t\left(y_{s}\right)\right]=-2 p_{11} \log \left[\frac{y_{2} p_{11}+p_{8} t\left(y_{s}\right)}{\sqrt{p_{8}}}\right] / \sqrt{p_{8}}-\left|z_{j}\right| \pi .
$$

Till now, the analytical solution of (14) has been found, and the flow diagram of the procedure is shown in Figure 4.

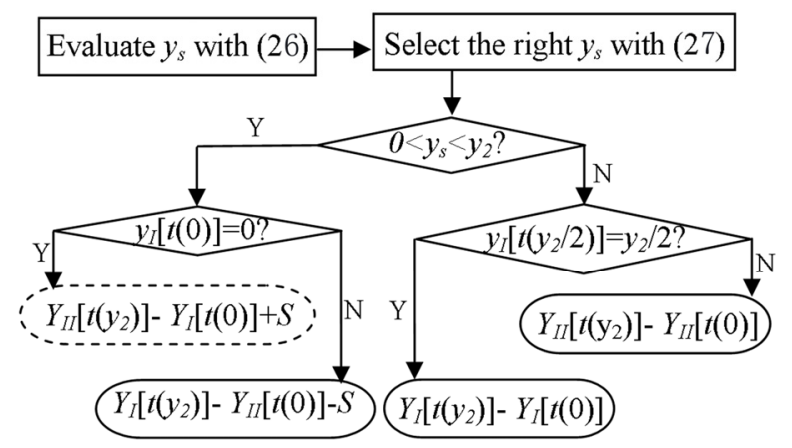

Figure 4: Flow diagram for analytically evaluating the integrals shown in (11) and (12). (The branch in the dashed frame was never accessed in calculation.).

The other integral of this type is

$$
\int_{0}^{y_{2}} \log \left(\frac{x_{2}}{y_{2}} y-x_{j}+\sqrt{\frac{p_{7}}{y_{2}^{2}} y^{2}+\frac{2 p_{9}}{y_{2}} y+p_{14}}\right) d y,
$$

and its analytical solution can also be obtained through the above process. The integrals represented by (12) can be analytically integrated using the same approach, and those represented by (13) can be analytically integrated by merely running the Matlab function int(). For the concision of the paper, their detailed integrations are omitted. 


\section{Calculation test}

As all the analytical solutions for the integrals in (5) and (6) are obtained with the aid of the computer, and the rectification method are gotten through calculation trials, it is necessary to justify the final analytical solutions. For this purpose, a testing calculation is carried out on a linearly interpolated triangular element using both analytical integration and numerical integration, and the analytical solutions can be justified if the result discrepancy between the two methods is trivial. In this testing calculation, the four integrals appearing in (4) are calculated at the two points $\mathrm{P}_{1}(1.2,0.4,0.05)$ and $\mathrm{P}_{2}(0.4,1.2,8)$, and the parameters used in the calculation are shown below:

$$
x_{2}=1.5 ; y_{2}=0.866 ; x_{3}=2 ; q_{1}=0.2 ; q_{2}=0.5 ; q_{3}=-0.2 \text {. }
$$

The numerical method employed here is the Riemann Sum, i.e., discretize the element in Figure 4 into micro squares of the size $\Delta x \times \Delta y$, and calculate the numerical integrals of $\mathrm{u}_{j}$ and $D_{j x}, D_{j y}, D_{j z}$ in (4) with the following formulae:

$$
\left\{\begin{array}{c}
\tilde{u}_{j}=\sum_{\mathrm{k}}\left(a^{q}+b^{q} x_{k}+c^{q} y_{k}\right) \frac{1}{4 \pi r_{k}} \Delta x \Delta y \\
\tilde{D}_{j x}=\sum_{\mathrm{k}}\left(a^{q}+b^{q} x_{k}+c^{q} y_{k}\right) \frac{x_{j}-x_{k}}{4 \pi r_{k}^{3}} \Delta x \Delta y \\
\tilde{D}_{j y}=\sum_{\mathrm{k}}\left(a^{q}+b^{q} x_{k}+c^{q} y_{k}\right) \frac{y_{j}-x_{k}}{4 \pi r_{k}^{3}} \Delta x \Delta y \\
\tilde{D}_{j z}=\sum_{\mathrm{k}}\left(a^{q}+b^{q} x_{k}+c^{q} y_{k}\right) \frac{z_{j}}{4 \pi r_{k}^{3}} \Delta x \Delta y
\end{array} .\right.
$$

In this testing calculation, $\Delta x$ and $\Delta y$ both take the value of $10^{-3}$ such that there are up to 865567 micro squares in the element. The element is so finely discretized as to guarantee the high accuracy of the numerical method, which serves as a benchmark for the analytical solution. The comparison is listed in Table 1, where the relative error is given in the form of percentage.

Table 1: Comparison between the numerical results and the analytical results.

\begin{tabular}{|l|l|l|l|l|}
\hline \multicolumn{2}{|c|}{} & Numerical result & Analytical result & $\begin{array}{l}\text { Relative } \\
\text { error(\%) }\end{array}$ \\
\hline \multirow{2}{*}{$U$} & $P_{1}$ & 0.04297488236604453 & 0.0429854239534878 & 0.0245 \\
\cline { 2 - 5 } & $P_{2}$ & 0.00142200559008392 & 0.00142263990124610 & 0.0446 \\
\hline \multirow{2}{*}{$D x$} & $P_{1}$ & 0.00984781774093461 & 0.00983102911646729 & 0.1708 \\
\cline { 2 - 5 } & $P_{2}$ & -0.0000141673524415 & -0.0000141785552194 & 0.0790 \\
\hline \multirow{2}{*}{$D_{y}$} & $P_{1}$ & -0.0403967509492500 & -0.0404088953008056 & 0.0301 \\
\cline { 2 - 5 } & $P_{2}$ & 0.0000167519902640 & 0.00001675616918126 & 0.0249 \\
\hline \multirow{2}{*}{$D_{z}$} & $P_{1}$ & 0.1024778500083124 & 0.10247993596632136 & 0.0020 \\
\cline { 2 - 6 } & $P_{2}$ & 0.0001745278953599 & 0.00017460541972824 & 0.0444 \\
\hline
\end{tabular}


From Table 1, it can be seen that the relative errors between the analytical results and the numerical results are trivial enough to justify the analytical solutions.

\section{Conclusion}

For IBEM applied in three dimensional problems governed by the Laplace equation, the closed form of analytical solutions of the integrals over a linearly interpolated triangular element can be deduced using the Matlab Symbolic Math Toolbox accompanied by a few manual rectifications and integral techniques. As most parts of the analytical solutions are the automatic output of the Matlab command window, we are spared from the tedious manual deriving work, and the string processing functions in the Matlab Symbolic Math Toolbox make the simplification of the analytical expressions more effective. Testing calculation has proved the validity of the all the analytical solutions derived in this paper.

\section{Appendix A. Expressions of the symbols $p_{n}$}

Symbols bearing the mark '*' represent the rectified expressions.

$$
\begin{gathered}
p_{1}=\frac{\left[\left(z_{j}-y_{j} \mathrm{i}\right)\left(x_{3}-x_{2}\right)+\left(x_{3}-x_{j}\right) y_{2} \mathrm{i}\right]^{2}}{y_{2}^{2}} \\
p_{2}=-\frac{\left[\left(y_{j}+z_{j} \mathrm{i}\right) x_{2}-y_{2} x_{j}\right]^{2}}{y_{2}^{2}} \\
p_{3}=\frac{\left[\left(y_{j}+z_{j} \mathrm{i}\right) x_{2}^{2}-y_{2}\left(x_{j} x_{2}-y_{2} z_{j} \mathrm{i}\right)\right]\left(y_{2}-y_{j}-z_{j} \mathrm{i}\right)}{y_{2}^{2}} \\
p_{5}=\frac{p_{3}\left(y_{j}+z_{j} \mathrm{i}\right)}{\left(y_{2}-y_{j}-z_{j} \mathrm{i}\right)} \\
\left.p_{j} \mathrm{i}-\left(x_{3}-x_{j}\right)\left(x_{3}-x_{2}\right) y_{2}+\left(y_{j}+z_{j} \mathrm{i}\right)\left(x_{3}-x_{2}\right)^{2}\right]\left(y_{2}-y_{j}-z_{j} \mathrm{i}\right) \\
y_{2}^{2} \\
p_{7}=-\frac{p_{5}\left(y_{j}+z_{j} \mathrm{i}\right)}{\left(y_{2}-y_{j}-z_{j} \mathrm{i}\right)} \\
p_{7}=y_{2}^{2}+x_{2}^{2} \\
p_{9}=-\left(x_{j} x_{2}+y_{j} y_{2}\right) \\
p_{10}=-\left[\left(x_{3}-x_{j}\right)\left(x_{3}-x_{2}\right)+y_{j} y_{2}\right] \\
p_{11}=\left(x_{j}-x_{3}\right) y_{2}+\left(x_{3}-x_{2}\right) y_{j} \\
p_{12}=\left(x_{2}-x_{j}\right)^{2}+\left(y_{2}-y_{j}\right)^{2}+z_{j}^{2} \\
p_{13}\left(x_{3}-x_{j}\right)^{2}+y_{j}^{2}+z_{j}^{2}
\end{gathered}
$$




$$
\begin{aligned}
& p_{14}=x_{j}^{2}+y_{j}^{2}+z_{j}^{2} \\
& p_{15}=\operatorname{atanh}\left[\frac{p_{3}-p_{2}}{\sqrt{-p_{2}} \sqrt{p_{7}\left(y_{2}-y_{j}-z_{j} \mathrm{i}^{2} / y_{2}^{2}+2 p_{3}-p_{2}\right.}}\right] \\
& p_{16}=\operatorname{atanh}\left[\frac{p_{5}-p_{1}}{\sqrt{-p_{1}} \sqrt{p_{8}\left(y_{2}-y_{j}-z_{j} \mathrm{i}\right)^{2} / y_{2}^{2}+2 p_{5}-p_{1}}}\right] \\
& p_{17}=\operatorname{atanh}\left[\frac{p_{4}-p_{2}}{\sqrt{-p_{2}} \sqrt{p_{7}\left(y_{j}+z_{j} \mathrm{i}\right)^{2} / y_{2}^{2}+2 p_{4}-p_{2}}}\right] \\
& p_{18}=\operatorname{atanh}\left[\frac{p_{6}-p_{1}}{\sqrt{-p_{1}} \sqrt{p_{8}\left(y_{j}+z_{j} \mathrm{i}\right)^{2} / y_{2}^{2}+2 p_{6}-p_{1}}}\right] \\
& p_{15}^{*}= \begin{cases}p_{15} & \text { if } \operatorname{imag}\left(p_{15}\right) \geq 0 \\
p_{15}+\pi \mathrm{i} & \text { if } \operatorname{imag}\left(p_{15}\right)<0\end{cases} \\
& p_{16}^{*}= \begin{cases}p_{16} & \text { if } \operatorname{imag}\left(p_{16}\right) \geq 0 \\
p_{16}+\pi \mathrm{i} & \text { if } \operatorname{imag}\left(p_{16}\right)<0\end{cases} \\
& p_{17}^{*}= \begin{cases}p_{17} & \text { if } \operatorname{imag}\left(p_{17}\right) \geq 0 \\
p_{17}+\pi \mathrm{i} & \text { if } \operatorname{imag}\left(p_{17}\right)<0\end{cases} \\
& p_{18}^{*}= \begin{cases}p_{18} & \text { if } \operatorname{imag}\left(p_{18}\right) \geq 0 \\
p_{18}+\pi \mathrm{i} & \text { if } \operatorname{imag}\left(p_{18}\right)<0\end{cases} \\
& p_{19}=\log \left[\frac{p_{7}+p_{9}}{\sqrt{p_{7}}}+\sqrt{p_{12}}\right] \\
& p_{20}=\log \left[\frac{p_{10}+p_{8}}{\sqrt{p_{8}}}+\sqrt{p_{12}}\right] \\
& p_{21}=\log \left[\frac{p_{9}}{\sqrt{p_{7}}}+\sqrt{p_{14}}\right] \\
& p_{22}=\log \left[\frac{p_{10}}{\sqrt{p_{8}}}+\sqrt{p_{13}}\right] \\
& p_{23}=\frac{\left(z_{j}-y_{j} \mathrm{i}\right) x_{2} / y_{2}+x_{j} \mathrm{i}}{\sqrt{-p_{2}}}\left(p_{15}^{*}-p_{17}^{*}\right) \\
& p_{24}=\frac{\left(z_{j}-y_{j} \mathrm{i}\right)\left(x_{3}-x_{2}\right) / y_{2}+\left(x_{3}-x_{j}\right) \mathrm{i}}{\sqrt{-p_{1}}}\left(p_{16}^{*}-p_{18}^{*}\right) \\
& p_{25}=p_{8} t^{2}+2 y_{2} p_{11} t-z_{j}^{2} y_{2}^{2} \\
& p_{26}=\log \left(\frac{p_{11} y_{2}+p_{8} t}{\sqrt{p_{8}}}+\sqrt{p_{25}}\right)
\end{aligned}
$$




\section{References}

[1] P. A. Martin, F. J. Rizzo, Hypersingular integrals: how smooth must the density be? International Journal for Numerical Methods in Engineering. 39 (1996) 687-704.

[2] Friedrich J, A linear analytical boundary element method (BEM) for 2D homogeneous potential problems, Computers \& Geosciences. 28 (2002) 679-692.

[3] Huanlin Zhou, Zhongrong Niu, Changzheng Cheng, Zhongwei Guan, Analytical integral algorithm applied to boundary layer effect and thin body effect in BEM for anisotropic potential problems, Computers and Structures. 86 (2008) 1656-1671.

[4] Huanlin Zhou, Zhongrong Niu, Changzheng Cheng, Zhongwei Guan, Analytical integral algorithm in the BEM for orthotropic potential problems of thin bodies. Engineering Analysis with Boundary Elements. 31 (2007) 739-748.

[5] Zhang X, An X, Exact integration and its application in adaptive boundary element analysis of two-dimensional potential problems, Communications in Numerical Methods in Engineering. 24 (2008) 1239-50.

[6] Jabisonski P, Integral and geometrical means in the analytical evaluation of the BEM integrals for a 3D Laplace equation, Engineering Analysis with Boundary Elements. 34 (2010) 264-273.

[7] Hayami K, Matsumoto H, A numerical quadrature for nearly singular boundary element integrals, Engineering Analysis with Boundary Elements. 13 (1994) 143-154.

[8] Xianyun Qin, Jianming Zhang, Guangyao Li, Xiaomin Sheng, Qiao Song, Donghui $\mathrm{Mu}$, An element implementation of the boundary face method for 3D potential problems, Engineering Analysis with Boundary Elements. 34 (2010) 934-943.

[9] Davey K, Hinduja S, Analytical integration of linear three-dimensional triangular elements in BEM, Appl Math Model. 13 (1989) 450-61.

[10] J. Milroy, S. Hinduja, K. Davey, The elastostatic three-dimensional boundary element method: analytical integration for linear isoparametric triangular elements, Appl Math Modelling. 21 (1997) 763-782.

[11] A. Salvadori, A. Temponi, Analytical integrations for the approximation of 3D hyperbolic scalar boundary integral equations. Engineering Analysis with Boundary Elements. 34 (2010) 977-994.

[12] A. Salvadori, Analytical integrations in 3D BEM for elliptic problems: evaluation and implementation, International Journal for Numerical Methods in Engineering. http://msvlab.hre.ntou.edu.tw/ijnme2010-SalvadinPress.pdf, last access date: 2011.06.30, DOI: 10.1002/nme.2906. 